\title{
Sex-Specific Association Between Iron Status and the Predicted 10-Year Risk for Atherosclerotic Cardiovascular Disease in Hypertensive Patients
}

\author{
Juan Zhou ${ }^{1,2} \cdot$ Rui Zhao $^{2} \cdot$ Dongxia Wang $^{2} \cdot$ Qin Gao $^{3} \cdot$ Dan Zhao $^{1} \cdot$ Binfa Ouyang $^{1} \cdot$ Liping Hao $^{2} \cdot$ Xiaolin Peng $^{1,4}$
}

Received: 4 August 2021 / Accepted: 10 October 2021 / Published online: 24 January 2022

(C) The Author(s) 2021

\begin{abstract}
Serum ferritin (SF) and haemoglobin $(\mathrm{Hb})$ are widely used in clinical practice to assess iron status. Studies exploring the relationship of SF and $\mathrm{Hb}$ with atherosclerotic cardiovascular disease (ASCVD) risk have yielded conflicting results, and some indicated sex specificity. Hypertensive patients have abnormal iron status. However, research on patients with hypertension is limited. We aim to investigate the sex-specific links of SF and $\mathrm{Hb}$ with the predicted 10-year ASCVD risk in hypertensive patients. This cross-sectional study included 718 hypertensive men and 708 hypertensive women. The predicted 10-year ASCVD risk was calculated based on the China-PAR equation. The dose-response curves were illustrated by fitting linear and quadratic models. In hypertensive men, the iron status fits for a quadratic model for ASCVD risk, showing a U-shape. After adjusting for potential confounding factors, the regression coefficients and $95 \%$ confidence intervals (95\% CI) across tertile of SF were 0.0 (reference), $-0.99(-1.65,-0.33)$ and $-0.22(-0.88,0.44)$, and of Hb were 0.0 (reference), -0.74 $(-1.41,-0.08)$ and $-0.77(-1.46,-0.08)$. In hypertensive women, iron status was linearly and positively associated with ASCVD risk. Per one unit increment of log-transformed SF as well as $\mathrm{Hb}$ was associated with a 1.22 (95\% CI: 0.97, 1.48) and 0.04 (95\% CI: 0.02, 0.07) increased in ASCVD risk score, respectively. A significant interaction between iron status and inflammation on ASCVD risk was observed in hypertensive women. SF and Hb showed a U-shape with ASCVD risk in hypertensive men; however, a positive linear relationship was observed in hypertensive women.
\end{abstract}

Keywords Iron $\cdot$ Ferritin $\cdot$ Haemoglobin $\cdot$ Atherosclerotic cardiovascular disease $\cdot$ Hypertension $\cdot$ Sex-specific

\section{Introduction}

Liping Hao and Xiaolin Peng contributed equally to this work.

Xiaolin Peng

xiaolinpeng@hotmail.com

1 Shenzhen Nanshan Center for Chronic Disease Control, Shenzhen 518051, China

2 Department of Nutrition and Food Hygiene, Hubei Key Laboratory of Food Nutrition and Safety, and Ministry of Education Key Laboratory of Environment and Health, School of Public Health, Tongji Medical College, Huazhong University of Science and Technology, Wuhan 430030, China

3 Department of Public Health, Jining Medical University, Jining 272067, China

4 Department of Oncology, Injury Prevention and Nutrition, Shenzhen Nanshan Center for Chronic Disease Control, 7 Huaming Road, Shenzhen 518051, China
Iron is an essential trace mineral that plays an irreplaceable role in the normal physiological processes of the body [1]. Iron deficiency (ID) is the leading cause of anaemia and is characterised by the insufficient synthesis of haemoglobin ( $\mathrm{Hb})$ [2]. Contrarily, free iron is highly reactive. To reduce oxidative damage, the iron that exceeds metabolic requirements is stored intracellularly as ferritin [3]. Consequently, the amount of iron deposited is closely reflected by the concentration of serum ferritin (SF). Clinically, $\mathrm{Hb}$ and SF are widely used as indicators of iron status [4].

The association between iron status and cardiovascular disease has been studied extensively since Sullivan proposed the iron-heart hypothesis [5], which suggested that increased iron deposits contribute to the risk of cardiovascular disease. Although several subsequent prospective studies backed up this theory, showing that elevated SF concentrations were linked to an increased risk of carotid atherosclerosis [6] and 
myocardial infarction (MI) [7], a huge body of epidemiological research has not confirmed this connection [8-12]. Furthermore, some surveys indicated that low iron status may also be an independent risk factor for coronary artery disease and stroke [13, 14], and with sex specificity [15]. For example, a large prospective cohort study in community-living adults found the U-shape association between $\mathrm{Hb}$ with stroke incidents only in women [15]. The sex-specific association between iron status and cardiovascular disease risk remains to be further explored.

Hypertension, a well-known contributor to atherosclerotic cardiovascular disease (ASCVD), is suggested to be related to abnormal iron status [16]. When compared to the nonhypertensive individuals, the SF and $\mathrm{Hb}$ levels are markedly higher in hypertensive patients [16]. Indeed, the perturbation of iron status is more pronounced when aortic stiffness is increased in hypertensive patients [17]. The previous study has noted that excess iron affects the insulin resistance state and sympathetic neural function in hypertensive patients [18], and both of which are highly related to ASCVD [19, 20]. Thus, the abnormal iron status may be a potential key factor in the development of ASCVD in hypertensive patients. However, information on the relationship between iron status and ASCVD risk in individuals with hypertension is extremely limited.

Clinically, the composite risk score is the standard approach for predicting future ASCVD risk, determining the need for further testing and initiating therapeutic intervention [21]. The predicted 10-year ASCVD risk, derived from the Prediction model for ASCVD risk in China (China-PAR equations), represents the preclinical stage of comprehensive ASCVD risk and can guide public health and clinical practice [22]. Few studies had looked at the relationship between iron status and the predicted 10-year ASCVD risk.

In this context, we aimed to explore the sex-specific association of iron status with the predicted 10-year ASCVD risk in a cross-sectional study comprising 718 hypertensive men and 708 hypertensive women from Shenzhen, China.

\section{Methods}

\section{Study Population}

In a cross-sectional study, we randomly recruited 1992 hypertensive patients at 53 community health service centres in the Nanshan District, Shenzhen, China, during 2017. These patients had been diagnosed with hypertension by the clinical physicians prior to the survey. As the ChinaPAR model was developed in a population aged $35-74$ years and without a history of ASCVD [22], we excluded individuals aged $<35$ years $(n=23)$ and aged $>74$ years $(n=170)$, as well as those patients with pre-existing clinical cardiovascular disease, including stroke, coronary heart disease and heart failure $(n=228)$. Individuals with selfreported kidney and liver disorder $(n=21)$, cancer $(n=14)$ and with an estimated glomerular filtration rate $\leq 60 \mathrm{~mL} /$ $\min / 1.73 \mathrm{~m}^{2}(n=61)$ and alanine aminotransferase levels $>3$ times of the upper normal limit $(n=5)$ were also excluded, as these patients possibility had abnormal iron status. After excluding those with missing data on ASCVD risk score $(n=44), 1426$ patients (718 men and 708 women) were eventually enrolled in this study (Fig. S1).

The survey was approved by the Ethics Committee of the Shenzhen Nanshan Centre for Chronic Disease Control and was performed following the Declaration of Helsinki and its amendments. Written informed consent was received from all included patients.

\section{Data and Biospecimen Collection}

The questionnaire, anthropometric measurements and blood sample collection were all taken on the same day. Trained research staff obtain demographic information, personal and family medical history, personal medication history and lifestyle information through a standardised questionnaire. Anthropometric data, including height, weight and waist circumference (WC), were acquired by direct measurements. Body mass index (BMI) was calculated by dividing weight in kilograms by the squared value of height in metres. Blood pressure (BP) was measured twice in a sitting position with an automated BP instrument (Omron-705CP; Omron Corp., Tokyo, Japan).

Morning blood samples were collected for each participant who fasted for more than $10 \mathrm{~h}$. Total cholesterol (TC), triglycerides (TG), high-density lipoprotein cholesterol (HDL-C), low-density lipoprotein cholesterol (LDL-C), fasting blood glucose (FBG), high-sensitivity $\mathrm{C}$-reactive protein (hs-CRP), alanine aminotransferase and creatinine were determined by the automatic analyser (Hitachi 7080, Tokyo, Japan). SF was determined by Abbotti2000SR automatic chemiluminescence immunoassay. The concentrations of $\mathrm{Hb}$ were acquired on an automated Mindray BC-5800 analyser (Mindray, Shenzhen, China).

\section{Predicted 10-Year Risk for ASCVD}

The 10-year ASCVD risk prediction is a useful tool to assess the risk of developing a first ASCVD event, including nonfatal MI, coronary heart disease death, fatal or nonfatal stroke, within 10 years in people free of ASCVD [23]. The ChinaPAR prediction equation, derived from sex-specific Cox proportional hazard models, has been shown to outperform the Pooled Cohort Equations in predicting 10-year ASCVD risk in the Chinese population [22]. The China-PAR model 
includes the following variables: sex, age, geographic region (northern/southern), urbanization (urban/rural), WC, TC, HDL-C, systolic BP (SBP), diastolic BP (DBP), treatment of hypertension (yes/no), diabetes mellitus (yes/no), current smoker (yes/no), family history of ASCVD (yes/no) and the interaction terms for age with risk factors that met predetermined statistical criteria. All continuous variables included in the equations were converted to natural logarithms and the categorical variables were expressed as 1 or 0 . The equation form is expressed as:

The predicted $10-$ year risk of $\operatorname{ASCVD}(\%)=1-\mathrm{S}_{10} \mathrm{e}^{\left(\operatorname{Ind} \mathrm{X}^{\prime} \mathrm{B}-\mathrm{MeanX} \mathrm{X}^{\prime} \mathrm{B}\right)}$

For the equation, the $S_{10}$ is the baseline survival rate for ASCVD at 10 years. IndX' $B$ is the individual sum, which is defined as the sum of "coefficient $\times$ value". MeanX'B is the overall mean "coefficient $\times$ value". The sex-specific values of $S_{10}$ and the coefficient, as well as the mean sum for the equation, were described in detail elsewhere [22].

\section{Ascertainment of Components of China-PAR and Covariates}

Marital status was divided into married and unmarried. Divorced, widowed and cohabiting were included as unmarried. Education level was classified into primary school or below, high school and college or above. The definition of the current smoker and diabetes mellitus has been previously defined [24]. Individuals who had an alcoholic drink at least once a week for more than 6 months were categorised as current drinkers. Physical activity was described as exercising at least three a week for at least 20 min each time over the last 6 months. Treatment of hypertension was identified as self-reported use of a prescription drug for hypertension during the past 2 weeks, control as $\mathrm{SBP}<140 \mathrm{~mm} \mathrm{Hg}$ and $\mathrm{DBP}<90 \mathrm{~mm} \mathrm{Hg}$. Based on the Chinese Guidelines on Prevention and Treatment of Dyslipidemia in Adults, the presence of abnormal serum lipid concentrations $(\mathrm{TC} \geq 6.2 \mathrm{mmo} / \mathrm{L}$ or $\mathrm{TG} \geq 2.3 \mathrm{mmo} / \mathrm{L}$ or LDL-C $\geq 4.1 \mathrm{mmo} / \mathrm{L}$ or HDL-C $<1.0 \mathrm{mmo} / \mathrm{L}$ ), or the use of lipid-lowering drugs within 2 weeks at the time of the survey were used to define dyslipidemia [25]. Family history of ASCVD was described as having at least a parent or a sibling with a stroke or MI [22]. Anaemia was defined as a $\mathrm{Hb}$ level below $130 \mathrm{~g} / \mathrm{L}$ for men and below $120 \mathrm{~g} / \mathrm{L}$ for women. ID was described as SF less than $30 \mathrm{ng} / \mathrm{mL}$ [26].

\section{Statistical Analysis}

In this study, SF and $\mathrm{Hb}$ were used to assess iron status. Relation between $\mathrm{SF}$ and $\mathrm{Hb}$ was tested using the Pearson test. Circulating ferritin concentration was right-skewed and therefore logarithmically transformed to approximate a normal distribution.

For descriptive purposes, we divided participants of each sex into three groups according to tertiles of iron status. We estimated means (SDs) and medians (interquartile ranges [IQRs]) for continuous variables and counts (percentages) for categorical variables. Linear regression for continuous variables (with the median concentration of each tertile as the variable used in the model) and $\chi^{2}$ with the linear-by-linear association test for categorical variables was used for linear trend tests across tertiles.

The generalised linear models were conducted to quantify the correlation of iron status (as a categorical variable) with the predicted 10-year ASCVD risk. As the results suggest a nonlinear quadratic relationship between iron status and 10-year ASCVD risk score in men (both $P$ for quadratic trend $<0.05)$. Consequently, we added a quadratic term for iron status in the multivariable-adjusted linear regression model for the predicted probability of future ASCVD risk and graphically presented the dose-response curve. If there is no evidence for a quadratic trend, we calculated the change in ASCVD risk score for each one-unit increase in the iron status with the use of linear regression models.

To control for potentially influential factors, we adjusted for marital status, education level, current drinker, physical activity, BMI and hs-CRP. The age and other variables were not included in the adjusted models as it is a component of the China-PAR equation. Adjusting for variables included in the China-PAR equation may result in over-adjustment. Inflammation affects levels of $\mathrm{SF}$ and $\mathrm{Hb}$ irrespective of iron status [27] and increases the risk of ASCVD [28]. To explore if the association between iron status and ASCVD risk was consistent across hs-CRP levels, we performed a subgroup analysis testing for interaction (hs-CRP $\times$ iron status) stratifying by median hs-CRP $(1.3 \mathrm{mg} / \mathrm{L})$.

All analyses were generated using $\mathrm{R}$ statistical software version 4.0.4. $P$-values below 0.05 indicate statistical significance.

\section{Results}

\section{Characteristics of Participants Stratified by Sex}

As presented in Table 1, compared to women, men had a higher proportion of married, highly educated, current smoker, current drinker and dyslipidaemia, and higher BMI, WC, SF, $\mathrm{Hb}$ and 10-year ASCVD score (all $P$-value $<0.05$ ). Besides, men were younger and had 
Table 1 Characteristics of the hypertensive population stratified by sex (Data are expressed as the means \pm SDs for normally distributed variables or as the median [interquartile range] for non-normally distributed variables, or counts (percentages) for categorical variables)

\begin{tabular}{|c|c|c|c|c|}
\hline Characteristics & Total $(n=1426)$ & Men $(n=718)$ & Women $(n=708)$ & $P$-value \\
\hline Age (years) & $56.15 \pm 9.64$ & $54.37 \pm 9.88$ & $57.96 \pm 9.05$ & $<0.001$ \\
\hline \multicolumn{5}{|l|}{ Marital status, $n(\%)$} \\
\hline Married & $1347(94.5)$ & $695(96.8)$ & $652(92.1)$ & \multirow[t]{2}{*}{$<0.001$} \\
\hline Unmarried & $79(5.5)$ & $23(3.2)$ & $56(7.9)$ & \\
\hline \multicolumn{5}{|l|}{ Education level, $n(\%)$} \\
\hline Primary school or below & $270(18.9)$ & $61(8.5)$ & $209(29.5)$ & \multirow[t]{3}{*}{$<0.001$} \\
\hline High school & $800(56.1)$ & $415(57.8)$ & $385(54.4)$ & \\
\hline College or above & $356(25.0)$ & $242(33.7)$ & $114(16.1)$ & \\
\hline \multicolumn{5}{|l|}{ Current smoker, $n(\%)$} \\
\hline No & $1223(85.8)$ & $519(72.3)$ & $704(99.4)$ & \multirow[t]{2}{*}{$<0.001$} \\
\hline Yes & $203(14.2)$ & $199(27.7)$ & $4(0.6)$ & \\
\hline \multicolumn{5}{|l|}{ Current drinker, $n(\%)$} \\
\hline No & 1009 (70.8) & $382(53.2)$ & 627 (88.6) & \multirow[t]{2}{*}{$<0.001$} \\
\hline Yes & $417(29.2)$ & $336(46.8)$ & $81(11.4)$ & \\
\hline \multicolumn{5}{|l|}{ Physical activity, $n(\%)$} \\
\hline No & $515(36.1)$ & $254(35.4)$ & $261(36.9)$ & \multirow[t]{2}{*}{0.596} \\
\hline Yes & $911(63.9)$ & $464(64.6)$ & $447(63.1)$ & \\
\hline \multicolumn{5}{|l|}{ Diabetes mellitus, $n(\%)$} \\
\hline No & $1131(79.3)$ & $578(80.5)$ & $553(78.1)$ & \multirow[t]{2}{*}{0.293} \\
\hline Yes & $295(20.7)$ & $140(19.5)$ & 155 (21.9) & \\
\hline \multicolumn{4}{|l|}{ Dyslipidaemia, $n(\%)$} & \multirow[t]{3}{*}{$<0.001$} \\
\hline No & $743(52.1)$ & $334(46.5)$ & $409(57.8)$ & \\
\hline Yes & $683(47.9)$ & $384(53.5)$ & $299(42.2)$ & \\
\hline \multicolumn{5}{|l|}{ Family history of ASCVD, $n(\%)$} \\
\hline No & $1126(79.0)$ & $577(80.4)$ & $549(77.5)$ & \multirow[t]{2}{*}{0.215} \\
\hline Yes & $300(21.0)$ & $141(19.6)$ & $159(22.5)$ & \\
\hline \multicolumn{5}{|l|}{ Treatment of hypertension, $n(\%)$} \\
\hline No & $379(26.6)$ & $188(26.2)$ & $191(27.0)$ & \multirow[t]{2}{*}{0.780} \\
\hline Yes & $1047(73.4)$ & $530(73.8)$ & $517(73.0)$ & \\
\hline \multicolumn{4}{|l|}{ Control of hypertension, $n(\%)$} & \multirow[t]{3}{*}{0.092} \\
\hline No & $562(39.4)$ & 299 (41.6) & $263(37.1)$ & \\
\hline Yes & $864(60.6)$ & $419(58.4)$ & $445(62.9)$ & \\
\hline \multicolumn{4}{|l|}{$\mathrm{ID}, n(\%)$} & \multirow[t]{3}{*}{$<0.001$} \\
\hline No & $1323(92.8)$ & $706(98.3)$ & $617(87.1)$ & \\
\hline Yes & $103(7.2)$ & $12(1.7)$ & $91(12.9)$ & \\
\hline \multicolumn{4}{|l|}{ Anaemia, $n(\%)$} & \multirow[t]{3}{*}{$<0.001$} \\
\hline No & $1232(86.4)$ & $647(90.1)$ & $585(82.6)$ & \\
\hline Yes & $194(13.6)$ & $71(9.9)$ & $123(17.4)$ & \\
\hline BMI $\left(\mathrm{kg} / \mathrm{m}^{2}\right)$ & $25.31 \pm 3.26$ & $25.88 \pm 3.13$ & $24.74 \pm 3.28$ & $<0.001$ \\
\hline $\mathrm{WC}(\mathrm{cm})$ & $87.84 \pm 9.40$ & $91.12 \pm 8.96$ & $84.52 \pm 8.65$ & $<0.001$ \\
\hline hs-CRP (mg/L) & $1.30[0.70,2.40]$ & $1.30[0.70,2.40]$ & $1.35[0.70,2.50]$ & 0.681 \\
\hline $\mathrm{SF}(\mathrm{ng} / \mathrm{mL})$ & $163.00[92.37,254.85]$ & $215.12[145.53,324.59]$ & $110.58[59.09,180.37]$ & $<0.001$ \\
\hline $\mathrm{Hb}(\mathrm{g} / \mathrm{L})$ & $136.98 \pm 13.86$ & $145.18 \pm 11.85$ & $128.67 \pm 10.36$ & $<0.001$ \\
\hline ASCVD risk score $(\%)$ & $5.30[3.20,7.80]$ & $5.70[3.50,8.20]$ & $4.90[3.08,7.50]$ & $<0.001$ \\
\hline
\end{tabular}

$A S C V D$, atherosclerotic cardiovascular disease; $B M I$, body mass index; $H b$, haemoglobin; $h s-C R P$, high sensitivity $\mathrm{C}$-reactive protein; $I D$, iron deficiency; $S F$, serum ferritin; $W C$, waist circumference

${ }^{a} P$-value was derived from Student's $t$-test and Mann-Whitney $U$ tests for continuous variables and chi-square tests for categorical variables 
a lower prevalence of ID and anaemia than women (all $P$-value $<0.05)$. There were no statistical differences between the sex in the distribution of physical activity (yes/no), diabetes mellitus (yes/no), family history of ASCVD (yes/no), treatment of hypertension (yes/ no), control of hypertension (yes/no) and hs-CRP (all $P$-value $>0.05$ ).

Figure 1 shows the distribution of the estimated 10 -year ASCVD risk score in the different subgroups. It was obvious that the predicted 10-year ASCVD risk was higher in the elderly, those with poorly controlled hypertension and those with diabetes $(P$-value $<0.05)$.

\section{Characteristics of Participants According to the Tertiles of Iron Status}

As shown in Table 2, men with higher SF levels had greater TG and hs-CRP and tended to be younger and current drinkers (all $P$ for trend $<0.05$ ). However, such a linear trend was not observed for the 10-year ASCVD risk score ( $P$ for trend $>0.05$ ). Additionally, the men in the highest tertile of $\mathrm{Hb}$ had greater BMI, TG and LDL-C, lower ASCVD risk score and tended to be younger (all $P$ for trend $<0.05$ ). As presented in Table 3, women with higher SF concentration had greater LDL-C, hs-CRP and 10-year ASCVD risk

\section{(A) Men}
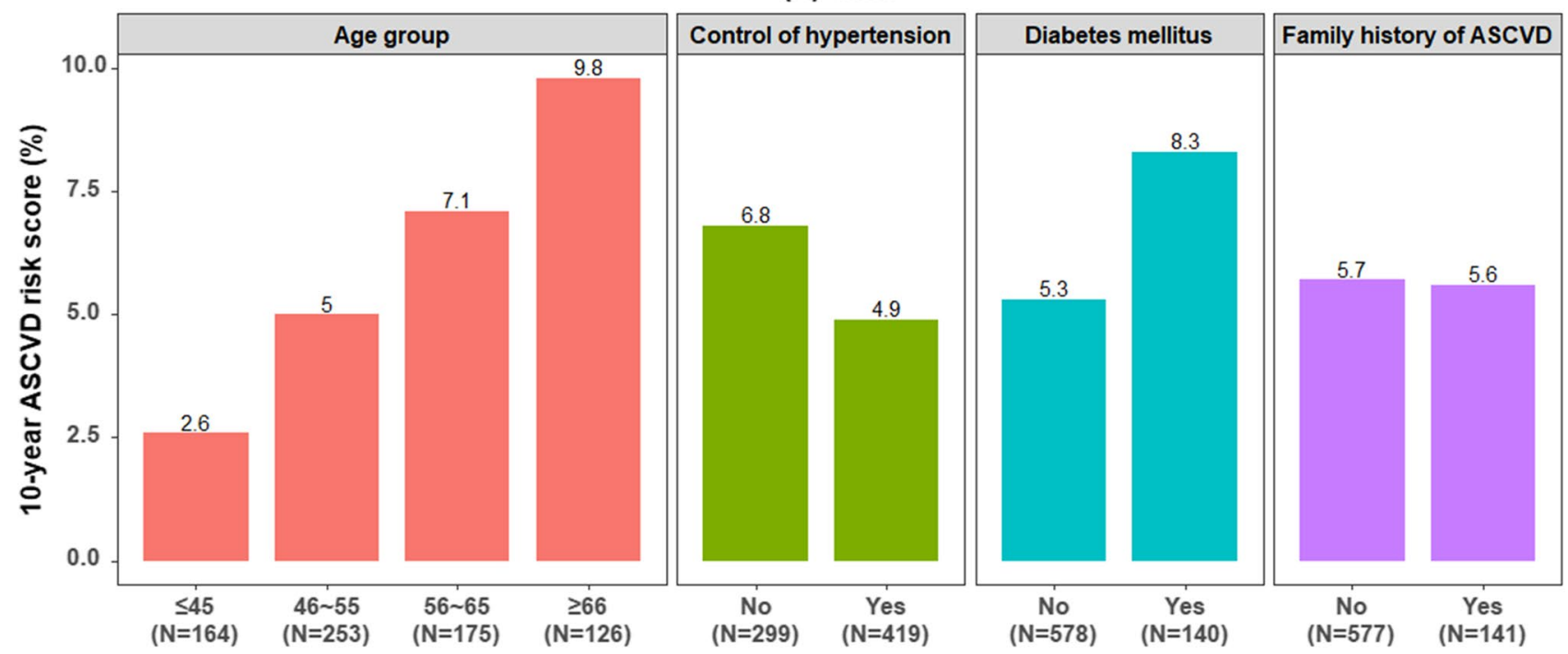

(B) Women
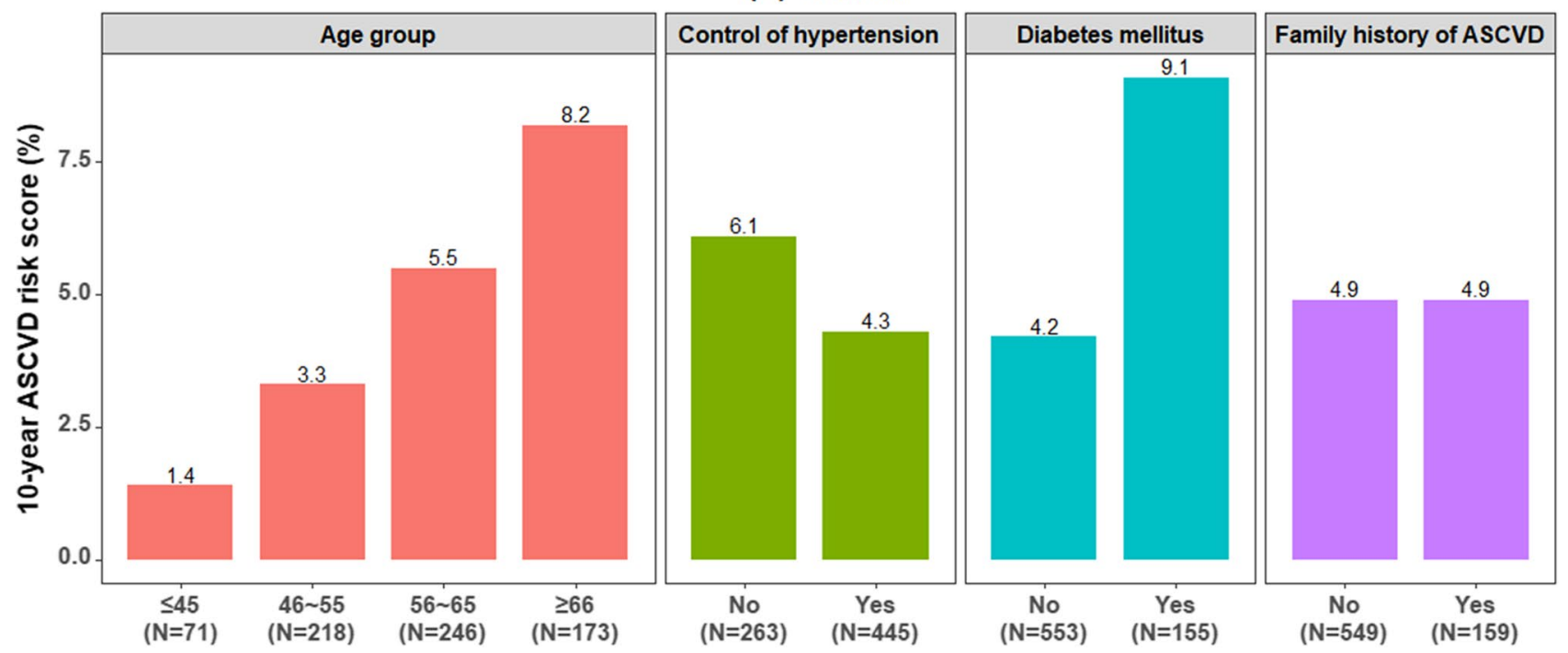

Fig. 1 A, B Distribution of the estimated 10-year ASCVD risk score in hypertensive patients, stratified by age group, control of hypertension, diabetes mellitus and family history of ASCVD. The $Y$-axis rep- resents the 10-year ASCVD risk score. ASCVD, atherosclerotic cardiovascular disease 
Table 2 Characteristics of the hypertensive population according to tertiles of iron status in men $(n=718)$ (Data are expressed as the means \pm SDs for normally distributed variables or as the median [interquartile range] for non-normally distributed variables, or counts (percentages) for categorical variables)

\begin{tabular}{|c|c|c|c|c|c|c|c|c|}
\hline \multirow[t]{2}{*}{ Characteristics } & \multicolumn{4}{|l|}{ Tertiles of SF } & \multicolumn{4}{|l|}{ Tertiles of $\mathrm{Hb}$} \\
\hline & $\mathrm{T} 1$ & $\mathrm{~T} 2$ & T3 & $P$ for trend ${ }^{\mathrm{a}}$ & $\mathrm{T} 1$ & $\mathrm{~T} 2$ & T3 & $P$ for trend \\
\hline Age (years) & $57.09 \pm 9.86$ & $53.18 \pm 9.91$ & $52.89 \pm 9.34$ & $<0.001$ & $57.77 \pm 10.24$ & $54.01 \pm 9.38$ & $51.60 \pm 9.15$ & $<0.001$ \\
\hline $\begin{array}{l}\text { Marital status, } \\
n(\%)\end{array}$ & & & & 0.602 & & & & 0.509 \\
\hline Married & 229 (96.6) & $235(96.3)$ & $231(97.5)$ & & 218 (98.6) & 246 (94.6) & $231(97.5)$ & \\
\hline Unmarried & $8(3.4)$ & $9(3.7)$ & $6(2.5)$ & & $3(1.4)$ & $14(5.4)$ & $6(2.5)$ & \\
\hline $\begin{array}{l}\text { Education } \\
\quad \text { level, } n(\%)\end{array}$ & & & & 0.004 & & & & 0.829 \\
\hline $\begin{array}{l}\text { Primary } \\
\text { school or } \\
\text { below }\end{array}$ & $29(12.2)$ & $23(9.4)$ & $9(3.8)$ & & $20(9.0)$ & $21(8.1)$ & $20(8.4)$ & \\
\hline High school & $127(53.6)$ & $135(55.3)$ & 153 (64.6) & & $127(57.5)$ & $149(57.3)$ & 139 (58.6) & \\
\hline $\begin{array}{l}\text { College or } \\
\text { above }\end{array}$ & $81(34.2)$ & $86(35.2)$ & 75 (31.6) & & $74(33.5)$ & $90(34.6)$ & 78 (32.9) & \\
\hline $\begin{array}{l}\text { Current } \\
\quad \text { drinker, } n(\%)\end{array}$ & & & & 0.008 & & & & 0.001 \\
\hline No & $140(59.1)$ & $131(53.7)$ & $111(46.8)$ & & $141(63.8)$ & $133(51.2)$ & 108 (45.6) & \\
\hline Yes & $97(40.9)$ & $113(46.3)$ & $126(53.2)$ & & $80(36.2)$ & $127(48.8)$ & $129(54.4)$ & \\
\hline $\begin{array}{l}\text { Physical activ- } \\
\text { ity, } n(\%)\end{array}$ & & & & 0.055 & & & & 0.017 \\
\hline No & $67(28.3)$ & $100(41.0)$ & 87 (36.7) & & $65(29.4)$ & $94(36.2)$ & $95(40.1)$ & \\
\hline Yes & $170(71.7)$ & $144(59.0)$ & $150(63.3)$ & & $156(70.6)$ & $166(63.8)$ & $142(59.9)$ & \\
\hline BMI $\left(\mathrm{kg} / \mathrm{m}^{2}\right)$ & $25.40 \pm 2.95$ & $26.16 \pm 3.23$ & $26.05 \pm 3.17$ & 0.050 & $24.93 \pm 2.99$ & $26.16 \pm 3.21$ & $26.44 \pm 2.98$ & $<0.001$ \\
\hline $\mathrm{TG}(\mathrm{mmol} / \mathrm{L})$ & $\begin{array}{l}1.43[0.95 \\
2.01]\end{array}$ & $\begin{array}{c}1.66[1.14, \\
2.36]\end{array}$ & $\begin{array}{l}1.84[1.46, \\
2.70]\end{array}$ & $<0.001$ & $\begin{array}{c}1.41[0.96, \\
1.96]\end{array}$ & $\begin{array}{l}1.73[1.20 \\
2.33]\end{array}$ & $\begin{array}{l}1.81[1.26 \\
2.56]\end{array}$ & $<0.001$ \\
\hline $\begin{array}{l}\text { LDL-C } \\
\qquad(\mathrm{mmol} / \mathrm{L})\end{array}$ & $3.20 \pm 0.74$ & $3.25 \pm 0.75$ & $3.17 \pm 0.74$ & 0.626 & $3.07 \pm 0.65$ & $3.24 \pm 0.81$ & $3.30 \pm 0.74$ & 0.002 \\
\hline hs-CRP (mg/L) & $\begin{array}{l}1.20[0.70, \\
2.10]\end{array}$ & $\begin{array}{l}1.30[0.70 \\
2.30]\end{array}$ & $\begin{array}{l}1.40[0.80, \\
2.90]\end{array}$ & 0.006 & $\begin{array}{l}1.30[0.70, \\
2.36]\end{array}$ & $\begin{array}{l}1.40[0.80, \\
2.52]\end{array}$ & $\begin{array}{l}1.20[0.70, \\
2.20]\end{array}$ & 0.062 \\
\hline $\mathrm{SF}$ (ng/mL) & $\begin{array}{c}117.01[85.57, \\
144.30]\end{array}$ & $\begin{array}{c}215.12 \\
{[190.69} \\
243.35]\end{array}$ & $\begin{array}{c}386.62 \\
{[325.71} \\
484.93]\end{array}$ & $<0.001$ & $\begin{array}{l}210.33 \\
{[128.45} \\
327.44]\end{array}$ & $\begin{array}{c}207.32 \\
\quad[140.01 \\
312.45]\end{array}$ & $\begin{array}{c}234.09 \\
{[165.02} \\
329.58]\end{array}$ & 0.010 \\
\hline $\mathrm{Hb}(\mathrm{g} / \mathrm{L})$ & $143.59 \pm 11.96$ & $146.34 \pm 11.47$ & $145.57 \pm 12.01$ & 0.133 & $131.65 \pm 6.91$ & $145.14 \pm 3.09$ & $157.84 \pm 6.29$ & $<0.001$ \\
\hline $\begin{array}{c}\text { ASCVD risk } \\
\text { score }(\%)\end{array}$ & $\begin{array}{l}6.50[4.40 \\
9.00]\end{array}$ & $\begin{array}{l}4.95[2.80 \\
7.70]\end{array}$ & $\begin{array}{l}5.60[3.50 \\
8.00]\end{array}$ & 0.242 & $\begin{array}{l}6.70[4.30 \\
9.40]\end{array}$ & $\begin{array}{l}5.45[3.58 \\
7.90]\end{array}$ & $\begin{array}{l}5.20[3.00, \\
7.60]\end{array}$ & 0.005 \\
\hline
\end{tabular}

$A S C V D$, atherosclerotic cardiovascular disease; $B M I$, body mass index; $H b$, haemoglobin; $h s-C R P$, high sensitivity C-reactive protein; $L D L-C$, low-density lipoprotein cholesterol; $S F$, serum ferritin; $T$, tertile; $T G$, triglyceride

${ }^{a}$ Linear trends were tested using linear regression for continuous variables (with the median levels of SF or $\mathrm{Hb}$ as a continuous variable included in the regression models), and $\chi^{2}$ with linear-by-linear association test for categorical variables

scores, and were more likely to be elder, unmarried and physically active (all $P$ for trend $<0.05$ ). Also, the women in the highest tertile of $\mathrm{Hb}$ had greater BMI, LDL-C, hsCRP and 10-year ASCVD risk score (all $P$ for trend $<0.05$ ). The distribution of the variables included in the China-PAR model stratified by tertiles of iron status in men and women is presented in Table $\mathrm{S} 1$ and Table S2, respectively.

Figure S2 illustrates the frequency histogram of log-transformed $\mathrm{SF}$, as well as the $\mathrm{Hb}$ concentration. Pearson $r$-test indicated that log-transformed SF was positively associated with $\mathrm{Hb}$ (men: $r=0.093, P=0.013$; women: $r=0.304, P<0.001$; Fig. S3).

\section{Association Between Iron Status and 10-Year ASCVD Risk Score}

Table 4 presents the association between iron status and 10 -year ASCVD risk score. For men, relative to the lowest 
Table 3 Characteristics of the hypertensive population according to tertiles of iron status in women $(n=708)$ (Data are expressed as the means \pm SDs for normally distributed variables or as the median [interquartile range] for non-normally distributed variables, or counts (percentages) for categorical variables)

\begin{tabular}{|c|c|c|c|c|c|c|c|c|}
\hline \multirow[t]{2}{*}{ Characteristics } & \multicolumn{4}{|l|}{ Tertiles of SF } & \multicolumn{4}{|l|}{ Tertiles of $\mathrm{Hb}$} \\
\hline & $\mathrm{T} 1$ & $\mathrm{~T} 2$ & T3 & $P$ for trend ${ }^{\mathrm{a}}$ & $\mathrm{T} 1$ & $\mathrm{~T} 2$ & $\mathrm{~T} 3$ & $P$ for trend ${ }^{\mathrm{a}}$ \\
\hline Age (years) & $52.32 \pm 9.37$ & $59.57 \pm 7.85$ & $61.94 \pm 6.85$ & $<0.001$ & $57.47 \pm 9.57$ & $58.00 \pm 8.72$ & $58.33 \pm 8.89$ & 0.305 \\
\hline $\begin{array}{l}\text { Marital status, } \\
n(\%)\end{array}$ & & & & 0.004 & & & & 0.214 \\
\hline Married & $225(96.2)$ & 219 (91.2) & 208 (88.9) & & $198(90.4)$ & $210(92.1)$ & $244(93.5)$ & \\
\hline Unmarried & $9(3.8)$ & $21(8.8)$ & $26(11.1)$ & & $21(9.6)$ & 18 (7.9) & $17(6.5)$ & \\
\hline $\begin{array}{l}\text { Education } \\
\quad \text { level, } n(\%)\end{array}$ & & & & 0.317 & & & & 0.527 \\
\hline $\begin{array}{l}\text { Primary } \\
\text { school or } \\
\text { below }\end{array}$ & $72(30.8)$ & $69(28.7)$ & $68(29.1)$ & & $66(30.1)$ & $66(28.9)$ & $77(29.5)$ & \\
\hline High school & $115(49.1)$ & $133(55.4)$ & 137 (58.5) & & $110(50.2)$ & $126(55.3)$ & $149(57.1)$ & \\
\hline $\begin{array}{l}\text { College or } \\
\text { above }\end{array}$ & $47(20.1)$ & $38(15.8)$ & $29(12.4)$ & & 43 (19.6) & $36(15.8)$ & $35(13.4)$ & \\
\hline $\begin{array}{l}\text { Current } \\
\quad \text { drinker, } n(\%)\end{array}$ & & & & 0.884 & & & & .0 .646 \\
\hline No & 207 ( 88.5) & $212(88.3)$ & 208 (88.9) & & 194 (88.6) & 205 (89.9) & 228 (87.4) & \\
\hline Yes & $27(11.5)$ & $28(11.7)$ & $26(11.1)$ & & $25(11.4)$ & $23(10.1)$ & $33(12.6)$ & \\
\hline $\begin{array}{l}\text { Physical activ- } \\
\text { ity, } n(\%)\end{array}$ & & & & 0.017 & & & & 0.097 \\
\hline No & $102(43.6)$ & $82(34.2)$ & 77 (32.9) & & $89(40.6)$ & $85(37.3)$ & 87 (33.3) & \\
\hline Yes & $132(56.4)$ & $158(65.8)$ & $157(67.1)$ & & $130(59.4)$ & 143 (62.7) & $174(66.7)$ & \\
\hline BMI $\left(\mathrm{kg} / \mathrm{m}^{2}\right)$ & $24.63 \pm 3.43$ & $24.63 \pm 3.04$ & $24.96 \pm 3.38$ & 0.238 & $24.50 \pm 3.55$ & $24.31 \pm 3.07$ & $25.31 \pm 3.16$ & 0.004 \\
\hline TG (mmol/L) & $\begin{array}{l}1.42[0.91, \\
1.92]\end{array}$ & $\begin{array}{l}1.41[1.05, \\
1.87]\end{array}$ & $\begin{array}{l}1.51[1.21, \\
2.07]\end{array}$ & 0.148 & $\begin{array}{c}1.41[0.97, \\
1.90]\end{array}$ & $\begin{array}{l}1.41[1.08, \\
1.91]\end{array}$ & $\begin{array}{l}1.50[1.12, \\
2.09]\end{array}$ & 0.165 \\
\hline $\begin{array}{l}\text { LDL-C } \\
\qquad(\mathrm{mmol} / \mathrm{L})\end{array}$ & $3.19 \pm 0.84$ & $3.32 \pm 0.73$ & $3.40 \pm 0.96$ & 0.008 & $3.13 \pm 0.75$ & $3.25 \pm 0.80$ & $3.50 \pm 0.93$ & $<0.001$ \\
\hline hs-CRP (mg/L) & $\begin{array}{l}1.10[0.70, \\
2.10]\end{array}$ & $\begin{array}{l}1.40[0.70, \\
2.60]\end{array}$ & $\begin{array}{l}1.60[0.80, \\
2.70]\end{array}$ & $<0.001$ & $\begin{array}{l}1.10[0.70, \\
2.43]\end{array}$ & $\begin{array}{l}1.30[0.70, \\
2.36]\end{array}$ & $\begin{array}{l}1.60[0.90, \\
2.60]\end{array}$ & 0.015 \\
\hline $\mathrm{SF}(\mathrm{ng} / \mathrm{mL})$ & $\begin{array}{l}41.98[21.42 \\
58.52]\end{array}$ & $\begin{array}{c}110.58 \text { [94.13, } \\
129.91]\end{array}$ & $\begin{array}{c}220.13 \\
{[181.73} \\
288.01]\end{array}$ & $<0.001$ & $\begin{array}{c}105.78[33.01, \\
170.93]\end{array}$ & $\begin{array}{c}106.08 \text { [62.74, } \\
161.12]\end{array}$ & $\begin{array}{c}128.79[79.83, \\
204.44]\end{array}$ & $<0.001$ \\
\hline $\mathrm{Hb}(\mathrm{g} / \mathrm{L})$ & $125.85 \pm 11.26$ & $129.46 \pm 8.88$ & $130.69 \pm 10.26$ & $<0.001$ & $116.85 \pm 7.17$ & $128.51 \pm 2.31$ & $138.73 \pm 4.97$ & $<0.001$ \\
\hline $\begin{array}{c}\text { ASCVD risk } \\
\text { score }(\%)\end{array}$ & $\begin{array}{l}3.60[1.83, \\
5.50]\end{array}$ & $\begin{array}{l}5.20[3.30, \\
7.43]\end{array}$ & $\begin{array}{l}6.10[4.20, \\
8.90]\end{array}$ & $<0.001$ & $\begin{array}{l}4.60[2.75, \\
6.80]\end{array}$ & $\begin{array}{l}4.70[3.10 \\
7.50]\end{array}$ & $\begin{array}{l}5.30[3.30, \\
7.90]\end{array}$ & 0.020 \\
\hline
\end{tabular}

$A S C V D$, atherosclerotic cardiovascular disease; $B M I$, body mass index; $H b$, haemoglobin; $h s-C R P$, high sensitivity C-reactive protein; $L D L-C$, low-density lipoprotein cholesterol; $S F$, serum ferritin; $T$, tertile; $T G$, triglyceride

${ }^{a}$ Linear trends were tested using linear regression for continuous variables (with the median levels of SF or Hb as a continuous variable included in the regression models), and $\chi^{2}$ with linear-by-linear association test for categorical variables

tertile, the adjusted regression coefficients $(\beta)$ and $95 \%$ confidence interval $(95 \% \mathrm{CI})$ of the second and third tertile of SF were $-0.99(95 \% \mathrm{CI}:-1.65,-0.33)$ and -0.22 (95\% CI: $-0.88,0.44)$, and for $\mathrm{Hb}$ were $-0.74(95 \%$ CI: $-1.41,-0.08)$ and -0.77 (95\% CI: $-1.46,-0.08$ ), respectively. The dose-response curve in men seems to be U-shape (Fig. 2A and B), as the quadratic SF term, as well as quadratic $\mathrm{Hb}$ term, was significantly related to the 10-year ASCVD risk score (SF (2): $\beta$ : 10.04,
SE: $3.65, P$-value $=0.006 ; \mathrm{Hb}(2): \beta: 8.11, \mathrm{SE}: 3.66$, $P$-value $=0.027)$. Contrary to men, the 10 -year ASCVD risk score for women increased in a dose-dependent manner with increasing SF and $\mathrm{Hb}$ (Table 4), and the quadratic models were not fit for women (Fig. 1C and D). In the continuous analyses, per one unit increase of log-transformed $\mathrm{SF}$ as well as Hb was associated with a 1.22 (95\% CI: 0.97, $1.48)$ and 0.04 (95\% CI: 0.02, 0.07) increased in ASCVD risk score, respectively (Table 4). 


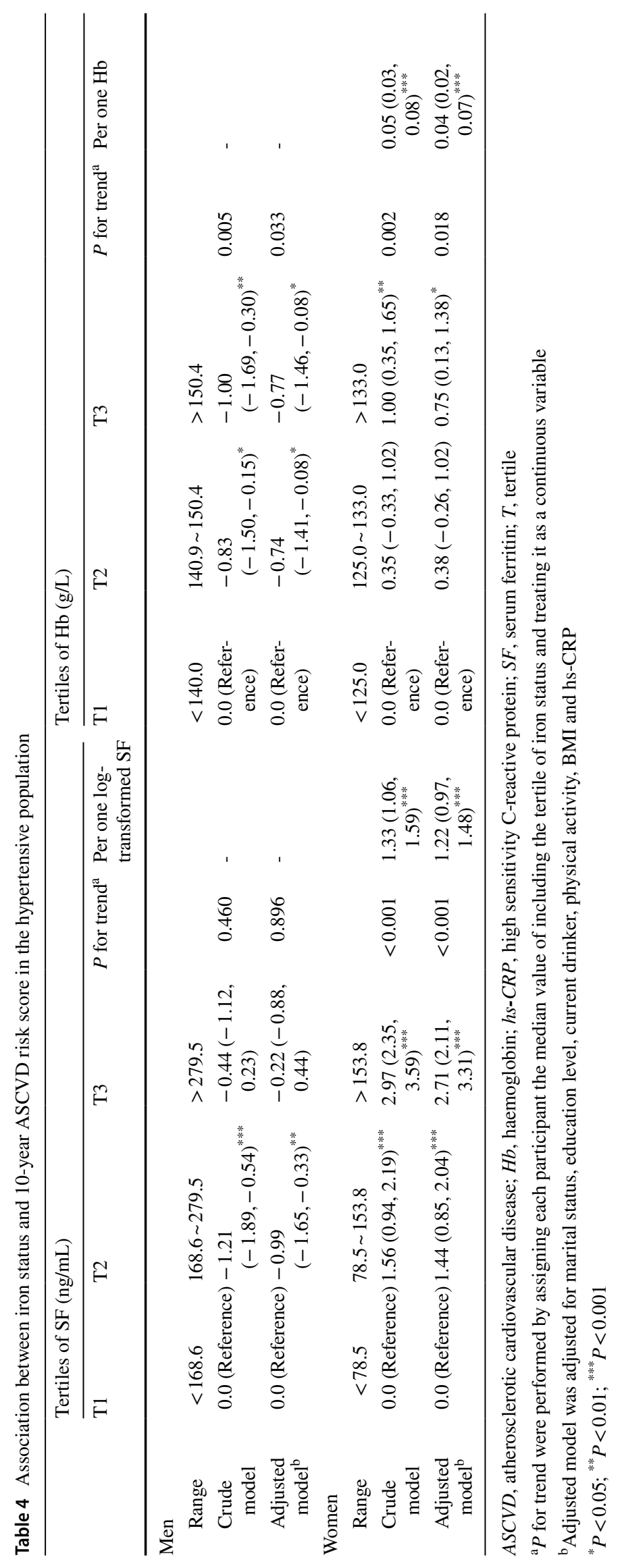


(A) Men

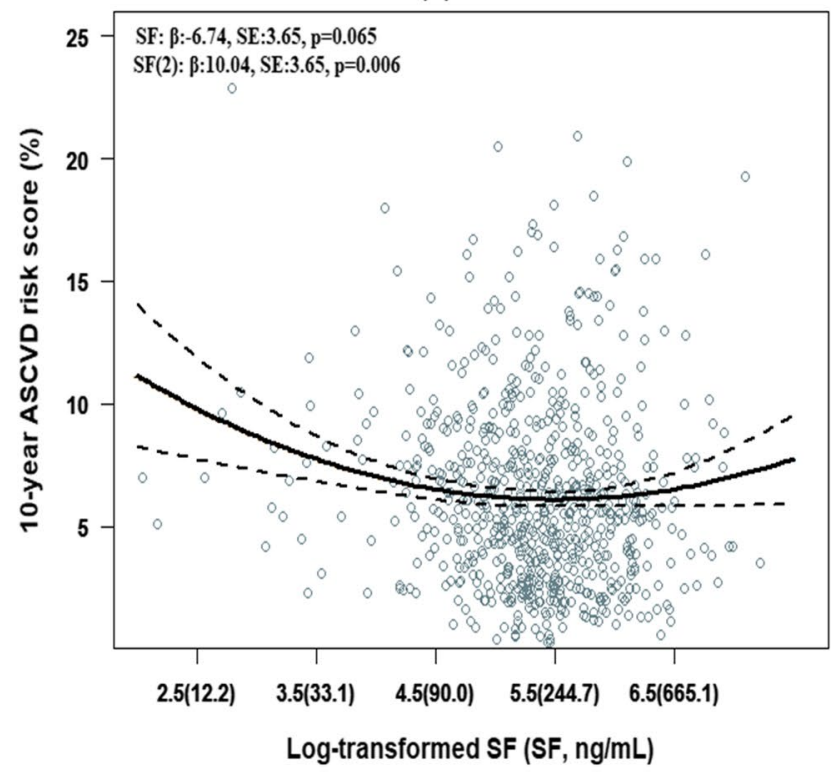

(C) Women

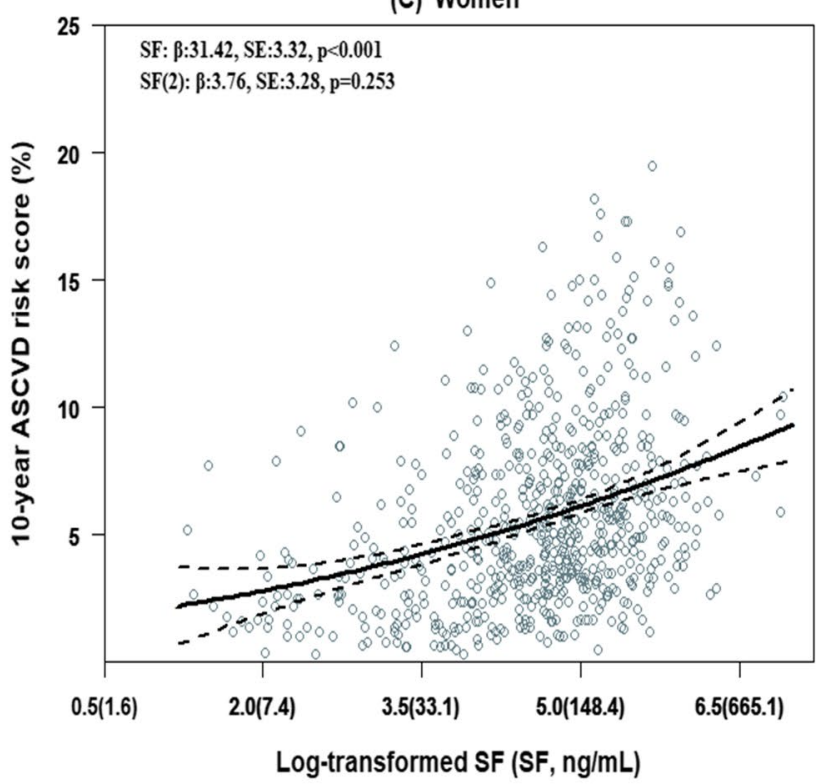

Fig. 2 A-D Predicted dose-response curves of 10-year ASCVD risk score by linear regression model in quadratic for iron status. The black solid lines correspond to the predicted line, and the black dotted lines corresponded to the confidence intervals. Models adjusted

\section{Interaction Between Iron Status and hs-CRP in Relation to 10-Year ASCVD Risk Score}

Participants were divided into two groups using the median hs-CRP as the cut-off point to explore the interaction between iron status and hs-CRP in relation to the 10-year ASCVD risk score. As shown in Table 5, in men, the nonlinear relationship between iron status and ASCVD risk
(B) Men

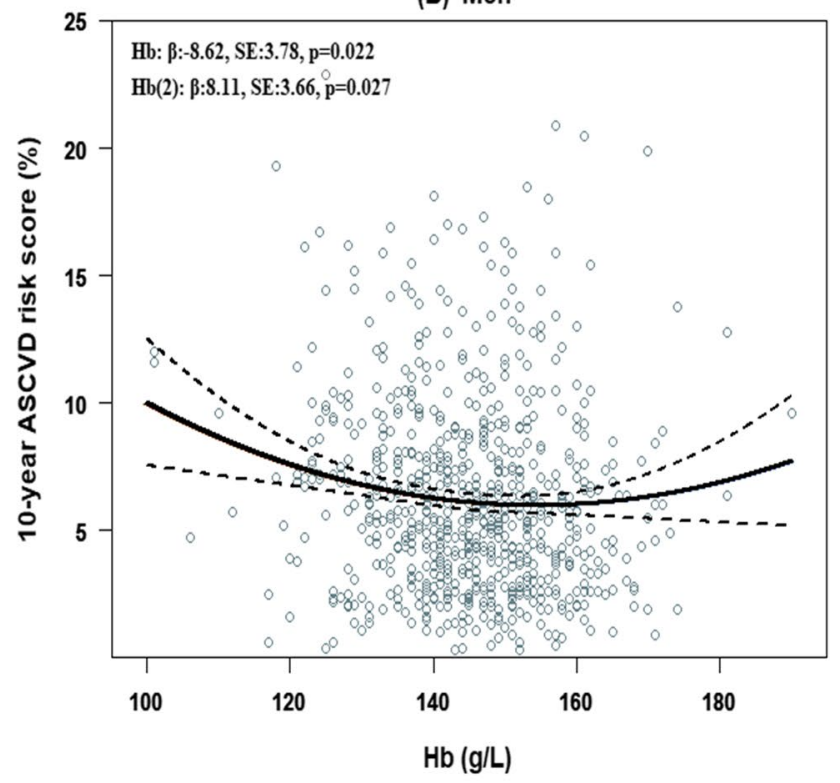

(D) Women

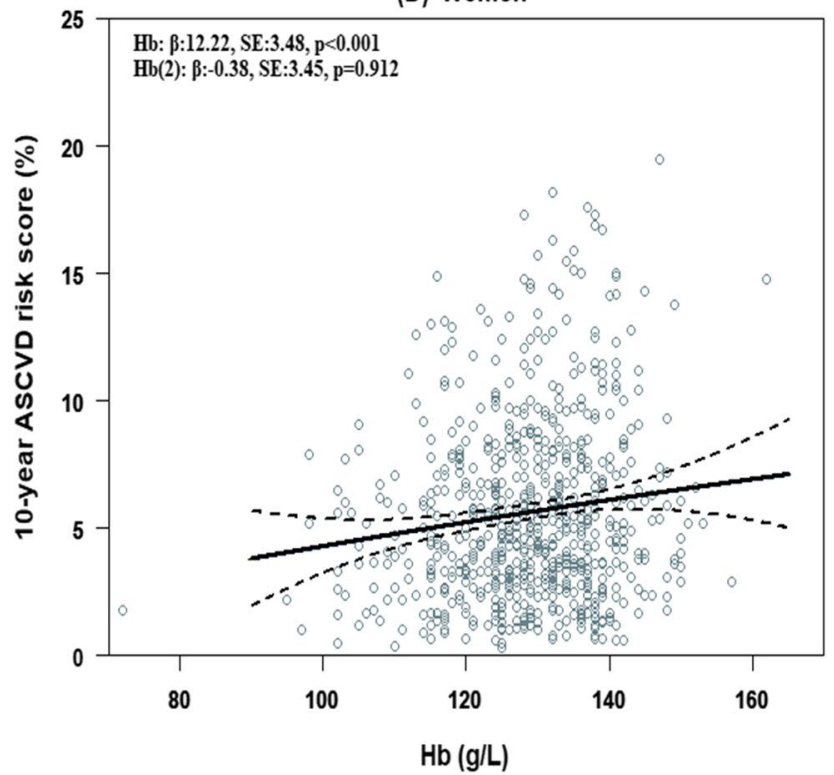

for marital status, education level, current drinker, physical activity, BMI and hs-CRP. ASCVD, atherosclerotic cardiovascular disease; $\mathrm{Hb}$, haemoglobin; SF, serum ferritin

score was consistent across hs-CRP strata. However, a significant interaction between hs-CRP and iron status in relation to ASCVD was observed in women $(P$ interaction for $\mathrm{SF}=0.004 ; P$ interaction for $\mathrm{Hb}=0.003$, Table 5). The associations of iron status with ASCVD risk were stronger for individuals with higher hs-CRP $(>1.3 \mathrm{mg} / \mathrm{L})$ than those with lower hs-CRP $(\leq 1.3 \mathrm{mg} / \mathrm{L})$. In hypertensive women with lower hs-CRP, the adjusted $\beta(95 \% \mathrm{CIs})$ from the lowest 


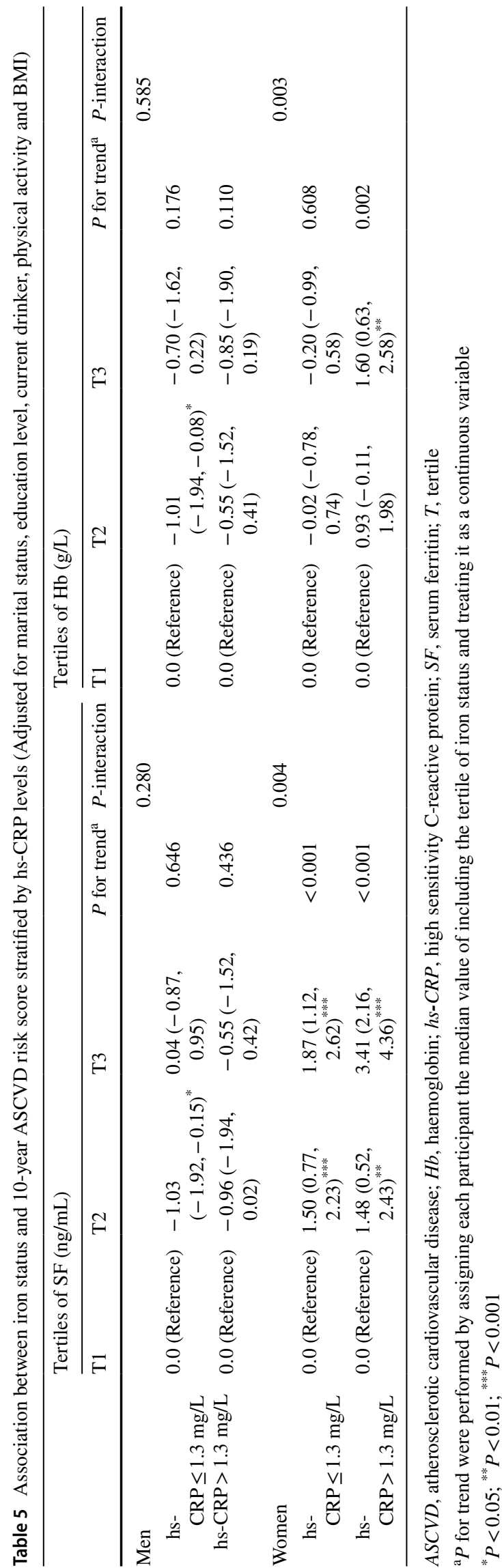

to the highest tertile of SF were $0.0,1.50(0.77,2.23)$ and $1.87(1.12,2.62)$, and of $\mathrm{Hb}$ were $0.0,-0.02(-0.78,0.74)$ and $-0.20(-0.99,0.58)$. In hypertensive women with higher hs-CRP, the adjusted $\beta$ (95\% CIs) from the lowest to the highest tertile of SF were $0.0,1.48(0.52,2.43)$ and $3.41(2.16,4.36)$, and of $\mathrm{Hb}$ were $0.0,0.93(-0.11,1.98)$ and $1.60(0.63,2.58)$.

\section{Discussion}

In this study that was performed on hypertensive patients, we found that iron status is strongly associated with the predicted 10-year ASCVD risk score and that the dose-effect curve varies by sex. Men showed a U-shaped curve, whereas women showed a linear positive correlation. Moreover, a significant interaction between iron status and hs-CRP in relation to the predicted ASCVD risk was observed in women.

Since the publication of the first study on the relationship between iron status and cardiovascular disease in 1981 [5], a rising number of epidemiological studies have discovered a link between high iron status and increased risk of ASCVD $[6,7,14,29]$. There are also experimental studies reporting the relationship between iron and the cardiovascular system, but they are not in accordance, e.g. iron decreased contractility in isolated rat cardiomyocytes [30] but there was not a relationship between contractility and iron in the heart in a whole animal model [31]. The Bruneck study, throughout a 5-year follow-up period, found a positive correlation between SF levels and ultrasound measures of carotid atherosclerosis progression [6]. Subsequently, Hb levels $\geq 170.0 \mathrm{~g} / \mathrm{L}$ was reported to be positively associated with increased risk for cardiac events in a cohort of US veterans [14]. Furthermore, the Mendelian randomization technique provided evidence that higher iron status, manifested as elevated SF concentration and transferrin saturation, is associated with increased stroke risk [29]. Notably, these studies primarily focused on the effects of iron on the risk of cardiovascular disease in the general population $[6,7]$. The existence of hypertension may perturb the iron-ASCVD association. Kim et al. recently indicated that the effect of $\mathrm{Hb}$ concentration on major adverse cardiovascular events was more pronounced in hypertensive patients, suggesting the need for in-depth studies in hypertensive populations [32]. Atherosclerosis is the main underlying pathology of ASCVD. According to Ross and Glomset's "Response to Injury" theory, atherosclerosis starts with endothelial cell injury [33]. It is now known that hypertension causes damage to the vascular endothelium [34]. On this basis, increased iron stores may further promote plaque formation through altered redox-sensitive signalling and inflammatory immune responses [35-37]. Some evidence suggested that iron overload can promote foam cell 
infiltration and necrotic core expansion, and thereby exacerbates the severity of atherosclerosis [38]. Furthermore, high $\mathrm{Hb}$ concentration can raise blood viscosity, which in turn increases peripheral resistance and decreases blood flow and perfusion [39]. Collectively, high iron may act synergistically with hypertension to promote atherosclerosis. The current study expands the body of knowledge by demonstrating a strong association between high iron status and increased ASCVD risk, highlighting the potential harms of high iron in hypertensive patients.

At the other end of the spectrum, ID may also have a detrimental effect on the cardiovascular outcome. The Atherosclerosis Risk in Communities study showed that individuals with ferritin levels below $30 \mathrm{ng} / \mathrm{mL}$ were at higher risk of heart failure compared to those with normal ferritin levels [40]. The results from the Ludwigshafen Risk and Cardiovascular Health Study showed an association between low iron status and cardiovascular as well as total mortality with an approximately 10-year follow-up [41]. Clinically, ID is more often considered to be an independent risk factor for poor cardiovascular prognosis. In the field of ASCVD, low iron status has not received equal attention as iron overload. However, a recent study found that ID-induced upregulation of transferrin levels and iron-binding ability can induce hypercoagulability by deactivating antithrombin through the thrombin/Factor XIIa complex [42]. At the same time, ID-induced thrombocytosis can increase the propensity for thrombosis [43]. Considering that ID affects the production of various antioxidant enzymes such as catalase and cytochrome oxidase, ID can also lead to oxidative damage and endothelial dysfunction [44]. Taken together, ID can be an etiological factor for ASCVD. In particular, Chang et al. identified a distinct correlation between prior ID and ischemic stroke in a case-control study comprising 204,372 individuals [45]. Contrary to the overwhelming evidence that anaemia and ID poses a significant risk to cardiovascular prognosis in patients with heart failure [46, 47], the clinical significance in hypertensive patients remains unclear. Only one study has pointed to anaemia as an important risk factor for cardiovascular and renal events in hypertensive patients [48]. This study adds to the literature evidence on the correlation between low iron status and ASCVD risk in hypertensive men.

Notably, despite the levels of iron were significantly lower in women than in men, we discovered that low iron status was related to an increased risk of ASCVD in men but not in women. Consistent with our study, the previous literature indicated that men with low iron status have a stronger association with ischaemic heart disease, stroke and a short lifespan than women with low iron status [49-51]. The possible explanation for the sex difference is numerous and requires further investigation. First, it is plausible that the repeated low iron status caused by heavy and continuous blood loss during menstruation may make the cardiovascular system of women more tolerant and resistant [52]. Second, the age-specific distribution of ID and anaemia probably has a major role in the sex difference associated with ASCVD risk. Men with anaemia and ID were predominantly older patients, while women were mainly younger subjects (Fig. S4). Therefore, younger age may be a factor explaining why low iron status is not associated with ASCVD risk score in female patients.

Inflammation affects iron regulation and is a recognised confounding factor [27]; we performed an interaction analysis of the iron status and inflammation. As a result, a stronger positive association between iron status and ASCVD risk was found in women with above-median hs-CRP relative to those with low hs-CRP. In response, we propose two biologically plausible explanations. The first explanation is that, as an acute-phase protein, the synthesis of ferritin is affected by inflammation and oxidative stress [53]. A review article has indicated that inflammatory cytokines, including the tumour necrosis factor $\alpha$, interleukin $1 \alpha$ and interleukin $1 \beta$, can upregulate ferritin expression [54]. The second explanation is that inflammation and oxidative stress affect the normal structure and function of erythrocytes. As noted in the literature, inflammation and oxidative stress induce ferri- and ferryl $\mathrm{Hb}$ production [55, 56] and the subsequent release of heme and iron triggers a chain reaction to the vascular system that is toxic and leads to the development of atherosclerosis $[57,58]$. The reason why the interaction was not found in men is unclear and still needs to be explored further.

This study is one of the few to explore the iron status and the predicted ASCVD risk in a hypertensive population. Hypertension may synergise with a disturbed iron status to promote the development of ASCVD. Therefore, the optimal range of iron under hypertension remains to be been determined. However, it should be admitted that there are several limitations. Firstly, we cannot establish the causal relation between iron status and the ASCVD risk, owing to the cross-sectional design. Secondly, the results were derived from Chinese hypertensive patients, which limits its applicability to other populations. Thirdly, although we excluded chronic diseases that may affect the iron status, and adjusted for several potential confounding factors, the unmeasured or incompletely measured factors may remain. Finally, we define SF less than $30 \mathrm{ng} / \mathrm{mL}$ as ID. Although prior research has confirmed its excellent positive predictive value for iron deficiency [59], chronic diseases and inflammation weaken its strength [26, 60]. Within our analysis, we included $\mathrm{Hb}$ as a way to mitigate the potential limitation. Nevertheless, further consideration of other iron parameters in the subsequent studies will be required to adequately resolve this concern. 
In conclusion, in the hypertensive patients, there was a sex-specific association between iron status and predicted 10-year ASCVD risk. A U-shape curve is suggested in hypertensive men, in which ASCVD risk is increased in the extreme lower and higher iron status. While for hypertensive women, ASCVD risk increased with increasing iron levels. Further work is required to confirm the effects of sex on the iron-ASCVD association, in particular, to elucidate the potential reasons for these findings.

Supplementary Information The online version contains supplementary material available at https://doi.org/10.1007/s12011-021-03060-y.

Acknowledgements The authors gratefully thank the community health service centres in the Nanshan District of Shenzhen for their hard work on blood sample collection. The authors also acknowledged the cooperation of the hypertensive individuals who participated in this study.

Author Contribution Juan Zhou, Xiaolin Peng and Liping Hao contributed to the conception or design of the work. Rui Zhao, Dongxia Wang, Qin Gao, Dan Zhao, Binfa Ooyang, Juan Zhou and Liping Hao contributed to the acquisition, analysis or interpretation of data for the work. Juan Zhou and Xiaolin Peng drafted the manuscript. Rui Zhao, Dongxia Wang, Qin Gao and Liping Hao critically revised the manuscript. All authors have read and approved the submission and the manuscript has not been published and is not being considered for publication elsewhere in whole or in part.

Funding This work was supported by the Sanming Project of Medicine in Shenzhen (grant numbers SZSM201803080); the Chinese Nutrition Society-Nutrition Research Foundation (grant numbers CNS-DSM2018A34); the Health and Family Commission of Shenzhen Municipality Funded Project (grant numbers SZSJ2017001); the Shenzhen Science and Technology Innovation Committee (grant numbers JCYJ20180302144310603); the Nanshan Science and Technology Innovation Bureau (grant numbers 2018039); and the Natural Science Foundation of Guangdong Province, China (grant numbers 2019A1515012026).

Availability of Data and Material The datasets generated and analysed during the current study are available from the corresponding author upon reasonable request.

\section{Declarations}

Ethics Approval The study was conducted in accordance with the Declaration of Helsinki, and the protocol was approved by the Ethics Committee of the Shenzhen Nanshan Centre for Chronic Disease Control.

Consent to Participate Signed informed consent was obtained from all participants.

Consent to Publish Not applicable.

Conflict of Interest The authors declare no competing interests.

Open Access This article is licensed under a Creative Commons Attribution 4.0 International License, which permits use, sharing, adaptation, distribution and reproduction in any medium or format, as long as you give appropriate credit to the original author(s) and the source, provide a link to the Creative Commons licence, and indicate if changes were made. The images or other third party material in this article are included in the article's Creative Commons licence, unless indicated otherwise in a credit line to the material. If material is not included in the article's Creative Commons licence and your intended use is not permitted by statutory regulation or exceeds the permitted use, you will need to obtain permission directly from the copyright holder. To view a copy of this licence, visit http://creativecommons.org/licenses/by/4.0/.

\section{References}

1. Crichton R (2016) The essential role of iron in biology. Iron Metabolism (4th ed):22-70. https://doi.org/10.1002/97811 18925645.ch2

2. Kassebaum NJ, Jasrasaria R, Naghavi M et al (2014) A systematic analysis of global anemia burden from 1990 to 2010 . Blood 123(5):615-624. https://doi.org/10.1182/blood-2013-06-508325

3. Zhang J, Chen X, Hong J et al (2021) Biochemistry of mammalian ferritins in the regulation of cellular iron homeostasis and oxidative responses. Sci China Life Sci 64(3):352-362. https:// doi.org/10.1007/s11427-020-1795-4

4. Restrepo-Gallego M, Díaz LE, Rondó PHC (2021) Classic and emergent indicators for the assessment of human iron status. Crit Rev Food Sci Nutr 61(17):2827-2840. https://doi.org/10. 1080/10408398.2020.1787326

5. Sullivan JL (1981) Iron and the sex difference in heart disease risk. Lancet 1(8233):1293-1294. https://doi.org/10.1016/s01406736(81)92463-6

6. Kiechl S, Willeit J, Egger G et al (1997) Body iron stores and the risk of carotid atherosclerosis: prospective results from the Bruneck study. Circulation 96(10):3300-3307. https://doi.org/ 10.1161/01.cir.96.10.3300

7. Salonen JT, Nyyssönen K, Korpela H et al (1992) High stored iron levels are associated with excess risk of myocardial infarction in eastern Finnish men. Circulation 86(3):803-811. https:// doi.org/10.1161/01.cir.86.3.803

8. Reyes C, Pons NA, Reñones CR et al (2020) Association between serum ferritin and acute coronary heart disease: a population-based cohort study. Atherosclerosis 293:69-74. https:// doi.org/10.1016/j.atherosclerosis.2019.12.011

9. Sun Q, Ma J, Rifai N et al (2008) Excessive body iron stores are not associated with risk of coronary heart disease in women. J Nutr 138(12):2436-2441. https://doi.org/10.3945/jn.108. 097766

10. Knuiman MW, Divitini ML, Olynyk JK et al (2003) Serum ferritin and cardiovascular disease: a 17-year follow-up study in Busselton, Western Australia. Am J Epidemiol 158(2):144-149. https://doi.org/10.1093/aje/kwg121

11. Shi Y, Zhou L, Huang LH et al (2011) Plasma ferritin levels, genetic variations in HFE gene, and coronary heart disease in Chinese: a case-control study. Atherosclerosis 218(2):386-390. https://doi.org/10.1016/j.atherosclerosis.2011.05.040

12. Ekblom K, Marklund SL, Jansson JH et al (2011) Iron stores and HFE genotypes are not related to increased risk of first-time myocardial infarction: a prospective nested case-referent study. Int J Cardiol 150(2):169-172. https://doi.org/10.1016/j.ijcard. 2010.04.001

13. Grammer TB, Kleber ME, Silbernagel G et al (2014) Hemoglobin, iron metabolism and angiographic coronary artery disease (The Ludwigshafen Risk and Cardiovascular Health Study). Atherosclerosis 236(2):292-300. https://doi.org/10. 1016/j.atherosclerosis.2014.07.002 
14. Chonchol M, Nielson C (2008) Hemoglobin levels and coronary artery disease. Am Heart J 155(3):494-498. https://doi.org/10. 1016/j.ahj.2007.10.031

15. Panwar B, Judd SE, Warnock DG et al (2016) Hemoglobin concentration and risk of incident stroke in community-living adults. Stroke 47(8):2017-2024. https://doi.org/10.1161/strok eaha.116.013077

16. Piperno A, Trombini P, Gelosa M et al (2002) Increased serum ferritin is common in men with essential hypertension. J Hypertens 20(8):1513-1518. https://doi.org/10.1097/00004872-20020 8000-00013

17. Valenti L, Maloberti A, Signorini S et al (2015) Iron stores, hepcidin, and aortic stiffness in individuals with hypertension. PLoS One 10(8):e0134635. https://doi.org/10.1371/journal. pone. 0134635

18. Seravalle G, Dell'Oro R, Quarti-Trevano F et al (2020) Sympathetic overactivation in patients with essential hypertension and hepatic iron overload. Hypertension 76(5):1444-1450. https://doi. org/10.1161/hypertensionaha.120.15511

19. Bornfeldt KE, Tabas I (2011) Insulin resistance, hyperglycemia, and atherosclerosis. Cell Metab 14(5):575-585. https://doi.org/ 10.1016/j.cmet.2011.07.015

20. Nardone M, Floras JS, Millar PJ (2020) Sympathetic neural modulation of arterial stiffness in humans. Am J Physiol Heart Circ Physiol 319(6):H1338-h1346. https://doi.org/10.1152/ajpheart. 00734.2020

21. Arnett DK, Blumenthal RS, Albert MA et al (2019) 2019 ACC/ AHA guideline on the primary prevention of cardiovascular disease: a report of the American College of Cardiology/American Heart Association Task Force on Clinical Practice Guidelines. Circulation 140(11):e596-e646. https://doi.org/10.1161/cir.00000 00000000678

22. Yang X, Li J, Hu D et al (2016) Predicting the 10-year risks of atherosclerotic cardiovascular disease in Chinese population: the China-PAR Project (Prediction for ASCVD Risk in China). Circulation 134(19):1430-1440. https://doi.org/10.1161/circulationaha. 116.022367

23. Goff DC Jr, Lloyd-Jones DM, Bennett G et al (2014) 2013 ACC/ AHA guideline on the assessment of cardiovascular risk: a report of the American College of Cardiology/American Heart Association Task Force on Practice Guidelines. Circulation 129(25 Suppl 2):S49-73. https://doi.org/10.1161/01.cir.0000437741.48606.98

24. Zhou J, Wang N, Wang D, et al (2021) Interactive effects of serum ferritin and high sensitivity C-reactive protein on diabetes in hypertensive patients. J Trace Elem Med Biol:126824.https:// doi.org/10.1016/j.jtemb.2021.126824

25. Joint committee for guideline revision (2018) 2016 Chinese guidelines for the management of dyslipidemia in adults. J Geriatr Cardiol 15(1):1-29. https://doi.org/10.11909/j.issn.1671-5411.2018.01.011

26. Camaschella C (2015) Iron-deficiency anemia. N Engl J Med 372(19):1832-1843. https://doi.org/10.1056/NEJMra1401038

27. Yuan X, Gao Y, Zhou W et al (2020) Effect of the inflammatory response on serum indices of iron status in late pregnancy. J Trace Elem Med Biol 61:126516. https://doi.org/10.1016/j.jtemb.2020. 126516

28. Danesh J, Whincup P, Walker M et al (2000) Low grade inflammation and coronary heart disease: prospective study and updated meta-analyses. BMJ 321(7255):199-204. https://doi.org/10.1136/ bmj.321.7255.199

29. Gill D, Monori G, Tzoulaki I et al (2018) Iron status and risk of stroke. Stroke 49(12):2815-2821. https://doi.org/10.1161/strok eaha. 118.022701

30. Moreb J, Hershko C, Hasin Y (1988) Effects of acute iron loading on contractility and spontaneous beating rate of cultured rat myocardial cells. Basic Res Cardiol 83(4):360-368. https://doi. org/10.1007/bf02005821
31. Mladenka P, Zatloukalova L, Filipsky T et al (2013) Common biomarkers of oxidative stress do not reflect cardiovascular dys/function in rats. Biomed Pap Med Fac Univ Palacky Olomouc Czech Repub 157(2):146-152. https://doi.org/10.5507/bp.2013.033

32. Kim IS, Lee BK, Yang PS et al (2020) Sex-based approach for the clinical impact of polycythaemia on cardiovascular outcomes in the general population. Eur J Prev Cardiol. https://doi.org/10. 1093/eurjpc/zwaa071

33. Ross R, Glomset JA (1976) The pathogenesis of atherosclerosis (first of two parts). N Engl J Med 295(7):369-377. https://doi.org/ 10.1056/nejm197608122950707

34. Petrie JR, Guzik TJ, Touyz RM (2018) Diabetes, hypertension, and cardiovascular disease: clinical insights and vascular mechanisms. Can J Cardiol 34(5):575-584. https://doi.org/10. 1016/j.cjca.2017.12.005

35. Yuan XM, Li W (2008) Iron involvement in multiple signaling pathways of atherosclerosis: a revisited hypothesis. Curr Med Chem 15(21):2157-2172. https://doi.org/10.2174/0929867087 85747634

36. Kraml PJ, Klein RL, Huang Y et al (2005) Iron loading increases cholesterol accumulation and macrophage scavenger receptor I expression in THP-1 mononuclear phagocytes. Metabolism 54(4):453-459. https://doi.org/10.1016/j.metabol.2004.10.012

37. Vinchi F, Porto G, Simmelbauer A et al (2020) Atherosclerosis is aggravated by iron overload and ameliorated by dietary and pharmacological iron restriction. Eur Heart J 41(28):26812695. https://doi.org/10.1093/eurheartj/ehz112

38. Hu X, Cai X, Ma R et al (2019) Iron-load exacerbates the severity of atherosclerosis via inducing inflammation and enhancing the glycolysis in macrophages. J Cell Physiol 234(10):1879218800. https://doi.org/10.1002/jcp. 28518

39. Kershenovich S, Modiano M, Ewy GA (1992) Markedly decreased coronary blood flow in secondary polycythemia. Am Heart J 123(2):521-523. https://doi.org/10.1016/0002-8703(92) 90671-h

40. Silvestre OM, Gonçalves A, Nadruz W Jr et al (2017) Ferritin levels and risk of heart failure-the Atherosclerosis Risk in Communities Study. Eur J Heart Fail 19(3):340-347. https://doi.org/ 10.1002/ejhf.701

41. Grammer TB, Scharnagl H, Dressel A et al (2019) Iron metabolism, hepcidin, and mortality (the Ludwigshafen Risk and Cardiovascular Health Study). Clin Chem 65(7):849-861. https://doi.org/ 10.1373/clinchem.2018.297242

42. Tang X, Fang M, Cheng R et al (2020) Iron-deficiency and estrogen are associated with ischemic stroke by up-regulating transferrin to induce hypercoagulability. Circ Res 127(5):651-663. https://doi.org/10.1161/circresaha.119.316453

43. Jimenez K, Leitner F, Leitner A et al (2021) Iron deficiencyinduced thrombocytosis increases thrombotic tendency in rats. Haematologica 106(3):782-794. https://doi.org/10.3324/haema tol.2019.245092

44. CoghettoBaccin A, LauermanLazzaretti L, Duarte Martins Brandao V et al (2009) Oxidative stress in older patients with iron deficiency anaemia. J Nutr Health Aging 13(8):666-670. https:// doi.org/10.1007/s12603-009-0195-6

45. Chang YL, Hung SH, Ling W et al (2013) Association between ischemic stroke and iron-deficiency anemia: a population-based study. PLoS One 8(12):e82952. https://doi.org/10.1371/journal. pone. 0082952

46. Anand IS, Gupta P (2018) Anemia and iron deficiency in heart failure: current concepts and emerging therapies. Circulation 138(1):80-98. https://doi.org/10.1161/circulationaha.118.030099

47. Chopra VK, Anker SD (2020) Anaemia, iron deficiency and heart failure in 2020: facts and numbers. ESC Heart Fail 7(5):20072011. https://doi.org/10.1002/ehf2.12797 
48. Kim-Mitsuyama S, Soejima H, Yasuda O et al (2019) Anemia is an independent risk factor for cardiovascular and renal events in hypertensive outpatients with well-controlled blood pressure: a subgroup analysis of the ATTEMPT-CVD randomized trial. Hypertens Res 42(6):883-891. https://doi.org/10.1038/ s41440-019-0210-1

49. Coutinho JM, Zuurbier SM, Gaartman AE et al (2015) Association between anemia and cerebral venous thrombosis: case-control study. Stroke 46(10):2735-2740. https://doi.org/10.1161/strok eaha.115.009843

50. Kim MY, Jee SH, Yun JE et al (2013) Hemoglobin concentration and risk of cardiovascular disease in Korean men and women - the Korean heart study. J Korean Med Sci 28(9):1316-1322. https:// doi.org/10.3346/jkms.2013.28.9.1316

51. Ezekowitz JA, McAlister FA, Armstrong PW (2005) The interaction among sex, hemoglobin and outcomes in a specialty heart failure clinic. Can J Cardiol 21(2):165-171

52. Visagie M, Pearson KR, Purvis TE et al (2019) Greater anemia tolerance among hospitalized females compared to males. Transfusion 59(8):2551-2558. https://doi.org/10.1111/trf.15338

53. Recalcati S, Invernizzi P, Arosio P et al (2008) New functions for an iron storage protein: the role of ferritin in immunity and autoimmunity. J Autoimmun 30(1-2):84-89. https://doi.org/10. 1016/j.jaut.2007.11.003

54. Kernan KF, Carcillo JA (2017) Hyperferritinemia and inflammation. Int Immunol 29(9):401-409. https://doi.org/10.1093/intimm/ dxx031
55. Weiss SJ (1982) Neutrophil-mediated methemoglobin formation in the erythrocyte. The role of superoxide and hydrogen peroxide. J Biol Chem 257(6):2947-2953

56. Giulivi C, Davies KJ (1994) Hydrogen peroxide-mediated ferrylhemoglobin generation in vitro and in red blood cells. Methods Enzymol 231:490-496. https://doi.org/10.1016/0076-6879(94) 31032-7

57. Woollard KJ, Sturgeon S, Chin-Dusting JP et al (2009) Erythrocyte hemolysis and hemoglobin oxidation promote ferric chloride-induced vascular injury. J Biol Chem 284(19):13110-13118. https://doi.org/10.1074/jbc.M809095200

58. Potor L, Bányai E, Becs G et al (2013) Atherogenesis may involve the prooxidant and proinflammatory effects of ferryl hemoglobin. Oxid Med Cell Longev 2013:676425. https://doi.org/10.1155/ 2013/676425

59. Mast AE, Blinder MA, Gronowski AM et al (1998) Clinical utility of the soluble transferrin receptor and comparison with serum ferritin in several populations. Clin Chem 44(1):45-51

60. Pasricha SR, Tye-Din J, Muckenthaler MU et al (2021) Iron deficiency. Lancet 397(10270):233-248. https://doi.org/10.1016/ s0140-6736(20)32594-0

Publisher's Note Springer Nature remains neutral with regard to jurisdictional claims in published maps and institutional affiliations. 